\title{
45GHz/20kW gyrotron setup with automated output power control for ECR ion source
}

\author{
$\underline{\text { A.I. Tsvetkov }}^{1}$, A.G. Eremeev ${ }^{1}$, V.V. Kholoptsev ${ }^{1}$, M.Yu. Shmelev ${ }^{1}$, I.V. Plotnikov ${ }^{1}$, Yu. V. Bykov ${ }^{1}$, \\ E.A. Kopelovich ${ }^{1}$, A.Yu. Novikov", M.M. Troitskiy ${ }^{1}$, M.V. Kuznetsov', K.A. Zhurin ${ }^{1}$, A.P. Fokin ${ }^{1}$, \\ M.V. Morozkin ${ }^{1}$, M.Yu. Glyavin ${ }^{1,2}$, M.I. Bakulin ${ }^{2}$, G.G. Denisov ${ }^{1,2}$, E.A. Soluyanova ${ }^{2}$, E.M. Tai ${ }^{2}$ \\ ${ }^{1}$ Institute of Applied Physics RAS, Nizhny Novgorod, Russia, tsvetkov@ipfran.ru \\ ${ }^{2}$ GYCOM Ltd., Nizhny Novgorod, Russia
}

The $45 \mathrm{GHz} / 20 \mathrm{~kW}$ automated gyrotron setup described below is intended for use in FECRAL (Fourth generation ECR ion source with Advanced design in Lanzhou, China) superconducting ion source. The FECRAL was proposed by Institute of Modern Physics (Chinese Academy of Science) for using in next generation Heavy Ion Accelerator Facility (HIAF), which requests ion source capable of delivering $50 \mathrm{p \mu A}$ of ${ }^{238} \mathrm{U}^{34+}$ pulsed beam and $25 \mathrm{p} \mu \mathrm{A}$ of ${ }^{238} \mathrm{U}^{34+} \mathrm{CW}$ beam for injector of a superconducting heavy ion linac $[1,2]$. When used as a part of such facility, the microwave source must satisfy the very strong requirements both for output radiation parameters and for possibilities of system integration.

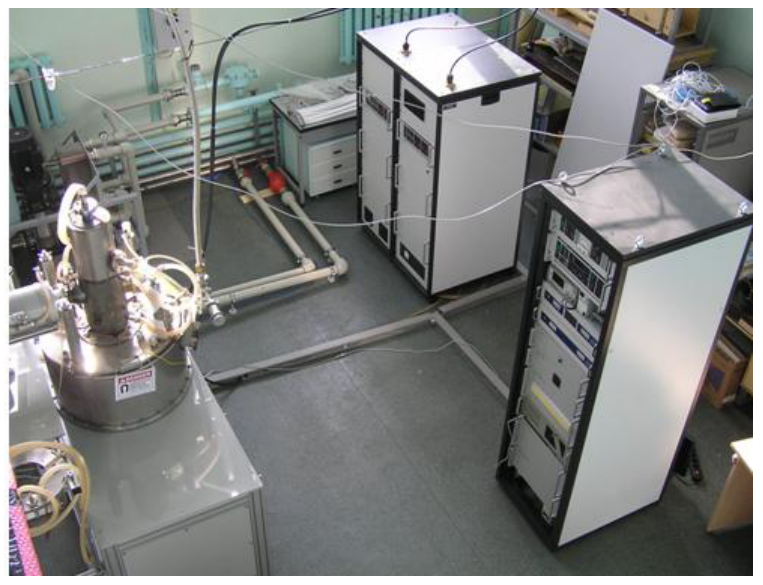

Fig. 1. The gyrotron setup general view

The gyrotron setup developed by IAP RAS jointly with GYCOM Ltd. [3] capable to provide $45 \mathrm{GHz}$ microwave radiation with output power from $1 \mathrm{~kW}$ up to 20 $\mathrm{kW}$ in both $\mathrm{CW}$ and pulse (5-200 ms with up to $10 \mathrm{~Hz}$ repetition rate) modes at $45 \mathrm{GHz}$ frequency.

The gyrotron tube is designed to operate with diodetype electron gun ( $\mathrm{LaB}_{6}$ cathode), and using of depressed collector for recuperation of the electron beam residual energy leads to considerable total efficiency increasing. Magnetic system is built on the basis of cryogen-free magnet JMTD-4T140 (Jastec Inc., Japan) with $140 \mathrm{~mm}$ warm bore diameter that allows the gyrotron to operate on fundamental harmonic ( $\mathrm{TE}_{6,3}$ mode).

The system allows triggering operation in pulse mode using external pulse generator as a reference. The transmission line consist of MOU, quasioptical elements, mode converter from the Gaussian beam $\mathrm{TEM}_{00}$ to $\mathrm{TE}_{01}$ or $\mathrm{TE}_{11}$ mode (at the user's option) and vacuum window. The last two mirrors and mode converter locate on the separate platform at the $2 \mathrm{~m}$ distance from the gyrotron, which results in $300 \mathrm{kV}$ electrical insulation, required for ECR ion source operation. The efficiency of the quasioptical transmission line is not less than $99 \%$ and the measured content of the $\mathrm{TE}_{01}$ or $\mathrm{TE}_{11}$ modes at the output of the mode converter more than $97 \%$ (Fig. 3).

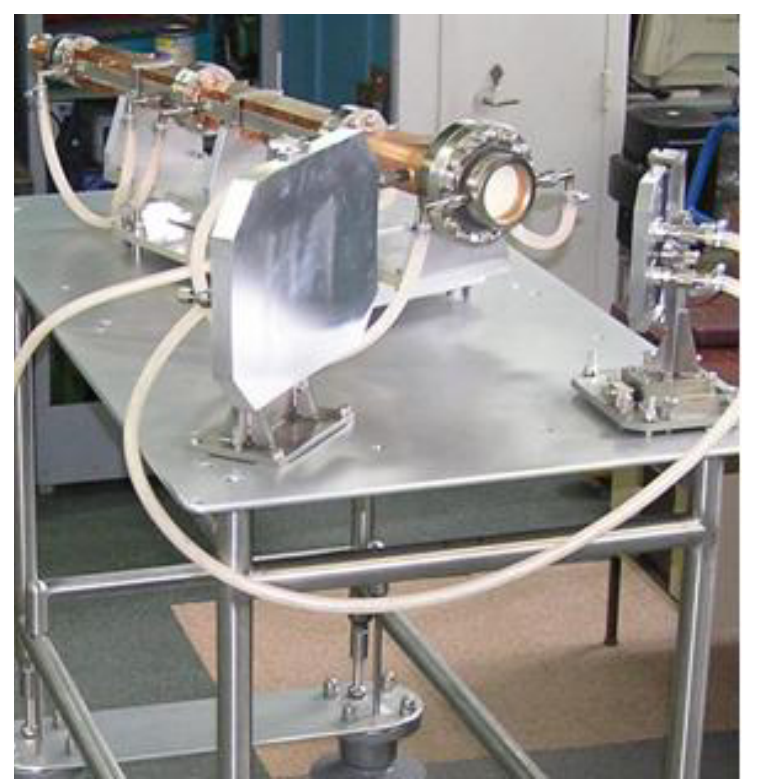

Fig. 2. Part of quasioptical transmission line: mirrors, mode converter from the Gaussian beam to $\mathrm{TE}_{01}$ or $\mathrm{TE}_{11}$ mode with vacuum window
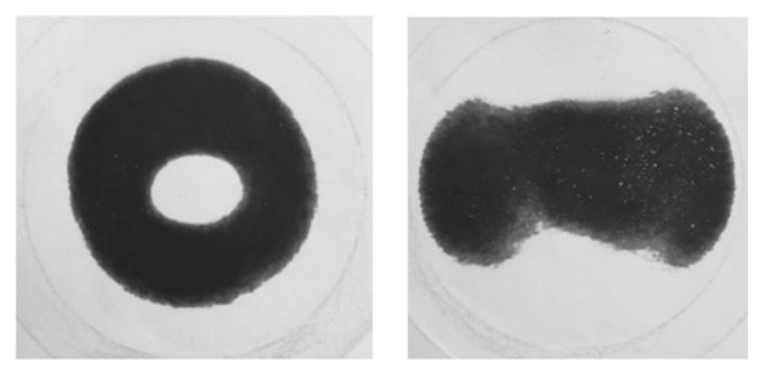

Fig. 3. MW radiation thermal prints at the output of the mode converter: left $-\mathrm{TE}_{01}$, right $-\mathrm{TE}_{11}$

The bi-directional couplers for monitoring and measuring the incident and reflected microwave power are mounted on the MOU mirrors. The attenuated microwave power for the incident and reflected waves is coupled to the rectangular waveguides through a set of the regular located circular holes of small diameter. An additional directional coupler is set in the channel of incident power. It divides the signal to 2 channels, one of them is connected to thermistor MW power meter and another is connected to MW detector. Signal of the reflected mi- 
crowave power goes to a microwave detector. The microwave detectors and power meter are situated outside the MOU and connected with directional couplers by flexible waveguides. Additional mirror (rotary switch) is situated between the main MOU mirrors and reflects the gyrotron radiation to the calorimetric dummy load.

The set of unique high-voltage power supplies (HVPS) made in IAP RAS provides synchronous operation to provide gyrotron operation at energy recovery regime (depressed collector) and ensure very short radiation power fall time (less than $1 \mu \mathrm{s}$ ) and rise time about 3 $\mu \mathrm{s}$ in pulse operation mode (Fig. 4) in the full range of output power levels. Cathode HVPS provides $25 \mathrm{kV} / 2.4$ A (negative polarity) output and anode HVPS provide 15 $\mathrm{kV} / 0.1$ A (positive polarity) output in $\mathrm{CW}$ and pulse modes. PID-regulation algorithm is used for stabilization of output power level using feedback signal from microwave power meter or semiconductor detector in $\mathrm{CW}$ or pulse mode.

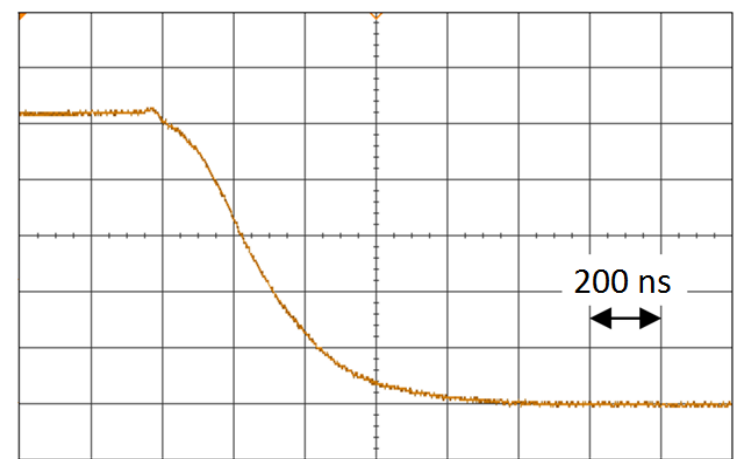

Fig. 4. Oscilloscope trace of the microwave power pulse falling edge

The setup is fully equipped with the automated control, data acquisition and safety interlock systems that guarantee convenient and trouble free remote and local operation. These systems provide starting up the setup and preparing it for operation follows a pre-set algorithm and automatic (full or partial) shutting out the power supplies in the event of failure or in emergency. The interrelation between operations of the setup individual devices is de- termined by the wiring of the relays without using any central processing unit or software. In order to provide fast switching off in the case of breakdown the signal from ARC-detector goes directly to cathode HVPS. Server software interacts with Data Acquisition System and passes information to the client software for displaying the actual state on the graphical user interface (Fig. 5). Possibility of integration into ion accelerator facility is achieved through the using of EPICS Channel Access network protocol-based interaction with server software.

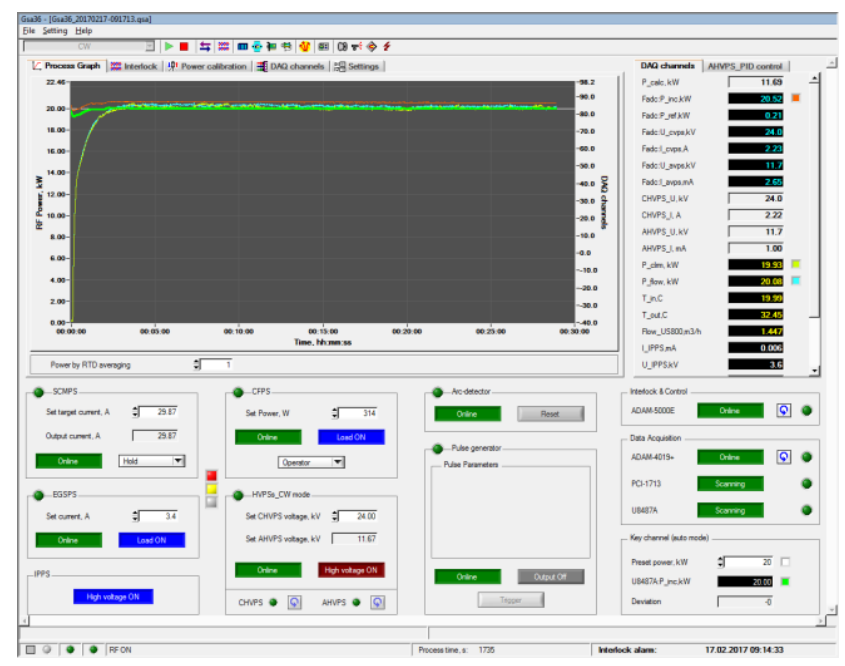

Fig. 5. The Client GUI displaying the results of microwave power control

\section{Acknowledgements}

Partially supported by the RF President Council for Grants, grant MK-3452.2017.8.

\section{References}

1. H. Zhao, L. Sun, J. Guo, W. Lu, Y. Yang, X. Zhang, Book of Abstracts: 16th Int. Conf. on Ion Sources, NY, USA, 2015, ThuPS26.

2. H. Zhao, L. Sun, The 22nd Int. Workshop on ECR Ion Sources, Busan, Korea, 2016, MOBO01.

3. G. Denisov, Yu. Bykov, M. Glyavin et. al., https:// doi.org/10.1109/PLASMA.2016.7533987. 